\title{
Síndrome de Rett: reporte de una nueva variante patogénica y revisión de la literatura a propósito de dos casos clínicos
}

\author{
Ana C. Jara-Ettinger ${ }^{1}$, Sabino Suárez-Hortiales ${ }^{1,2}$ y Oliver De la Torre-García ${ }^{1,2 *}$ \\ ${ }^{1}$ Unidad Médica de Alta Especialidad Hospital de Pediatría, Centro Médico Nacional Siglo XXI, Instituto Mexicano del Seguro Social; ${ }^{2}$ Centro \\ Médico Naval, Secretaría de Marina. Ciudad de México, México
}

\begin{abstract}
Resumen
Introducción: El síndrome de Rett es un trastorno del neurodesarrollo con una frecuencia estimada de 1/10,000 recién nacidos vivos, el cual se presenta con un modelo de herencia ligado al cromosoma X. Las variantes patogénicas en el gen MECP2, el cual codifica para una proteína que participa en el desarrollo y la diferenciación del sistema nervioso central, causan este síndrome. El objetivo de este trabajo fue describir dos casos de síndrome de Rett, uno de ellos con una nueva variante del gen MECP2. Casos clínicos: El primer caso se trata de una paciente de 5 años con microcefalia y regresión del neurodesarrollo desde los 3 años. Clínicamente se diagnosticó de síndrome de Rett en estadio III. Se realizó la secuenciación del gen MECP2 y se identificó una variante probablemente patogénica en estado heterocigoto, c.606delC (p. Thr203Argfs*7), que no ha sido reportada previamente. El segundo caso es una paciente de 17 años, referida por discapacidad intelectual grave, que se encontró clínicamente en estadio IV. Se realizó la secuenciación de MECP2 y se identificó una variante patogénica [c.880C>T(p.Arg294*)] ya descrita previamente. Conclusiones: El diagnóstico clínico de síndrome de Rett se llevó a cabo con criterios establecidos. La confirmación diagnóstica fue mediante la secuenciación de MECP2. Para el correcto abordaje de los trastornos del neurodesarrollo es primordial conocer el fenotipo de síndrome de Rett, asi como optar por el análisis molecular para la confirmación del diagnóstico. Los pacientes con síndrome de Rett requieren un seguimiento interdisciplinario para disminuir el impacto de las complicaciones.
\end{abstract}

Palabras clave: Síndrome de Rett. Autismo. Proteína de unión a CpG metilada (MECP2). Trastorno del neurodesarrollo.

\section{Rett syndrome: report of a new pathogenic variant and review of the literature regarding two clinical cases}

\begin{abstract}
Background: Rett syndrome is an X-linked neurodevelopmental disorder with an estimated frequency of 1/10,000 live births caused by hetereozygous pathogenic variants in the MECP2 gene, whose protein participates in the development and differentiation of the central nervous system. This study aimed to describe two cases with Rett syndrome diagnosis, one of them with a new variant of the MECP2 gene. Case reports: We first describe the case of a 5-year-old female with microcephaly and neurodevelopmental regression starting at 3 years old, clinically corresponding to stage III Rett syndrome. Sequencing of the MECP2 gene identified a heterozygous likely pathogenic variant [c.606delC (p.Thr203Argfs*7)] not reported previously.
\end{abstract}

Correspondencia:

*Oliver De La Torre-García

E-mail: olde5121@colorado.edu
Fecha de recepción: 06-05-2020

Fecha de aceptación: 31-07-2020

DOI: 10.24875/BMHIM.20000121
Disponible en internet: 23-07-2021

Bol Med Hosp Infant Mex. 2021;78(4):356-361

www.bmhim.com 1665-1146/@ 2020 Hospital Infantil de México Federico Gómez. Publicado por Permanyer. Este es un artículo open access bajo la licencia CC BY-NC-ND (http://creativecommons.org/licenses/by-nc-nd/4.0/). 
The second case is a 17-year-old female, referred due to severe intellectual disability, clinically found on stage IV. MECP2 sequencing was performed identifying a pathogenic variant previously described [C.880C > T (p.Arg294 *)]. Conclusions: Rett syndrome clinical diagnosis was carried out based on established criteria. MECP2 sequencing confirmed the diagnosis. For neurodevelopmental disorders approach, it is essential to know the phenotype of Rett syndrome and select the molecular tool for the diagnosis. Patients with Rett syndrome require interdisciplinary follow-up for reducing the impact of complications.

Keywords: Rett syndrome. Autism. Methyl-CpG binding protein (MECP2). Neurodevelopmental disorder.

\section{Introducción}

El síndrome de Rett (SR) es un trastorno del neurodesarrollo con modelo de herencia ligada al cromosoma $X$, que afecta casi exclusivamente al sexo femenino. La frecuencia estimada es de $1 / 10,000$ recién nacidos vivos y la mayoría de los casos son esporádicos, causados por mutaciones de novo. Este síndrome fue descrito por el médico Andreas Rett en la década de 1960, y en 1999 se descubrió que ciertas variantes patogénicas en MECP2 se asociaban a este síndrome $^{1,2}$. El gen MECP2 codifica para la proteína de unión a CpG metilada (MeCP2), que participa activamente en el desarrollo y la diferenciación neuronales ${ }^{3}$.

El SR es la causa más frecuente de discapacidad intelectual en el sexo femenino en todo el mundo. Aunque previamente se englobó dentro del espectro autista, hoy se reconocen claras diferencias. Si bien se presentan comportamientos similares a los del espectro autista, como conducta social anormal y falta de contacto visual durante la fase de regresión, después de este estadio y al aumentar la edad los pacientes con SR son más sociables y presentan mayor interacción visual $1^{4,5}$.

En el abordaje inicial de las pacientes de sexo femenino con sospecha de espectro autista es indispensable realizar en paralelo el diagnóstico clínico para SR, de acuerdo con los criterios establecidos por Neul et $a{ }^{6}$. Se clasifica en típico y atípico de la siguiente manera:

- Criterios para SR típico:

1. Periodo de regresión seguido por periodo de recuperación o estabilización.

2. Todos los criterios mayores y todos los criterios de exclusión.

3. No se requieren criterios de apoyo, a pesar de que con frecuencia están presentes.

- Criterios para SR atípico o variante:

1. Periodo de regresión seguido por periodo de recuperación o estabilización.

2. Al menos dos de los cuatro criterios mayores.

3. Cinco de los 11 criterios de apoyo.
- Criterios mayores:

1. Pérdida parcial o completa de habilidades manuales intencionales adquiridas.

2. Pérdida parcial o completa del lenguaje hablado adquirido.

3. Anormalidades de la marcha: deterioro (dispráxico) o ausencia de la capacidad.

4. Movimientos estereotípicos de la mano, como retorcer/apretar las manos, aplaudir/golpear, articular o lavar/frotar.

- Criterios de exclusión para SR típico:

1. Lesión cerebral secundaria a trauma (perinatal o posnatal), enfermedad neurometabólica o infección grave que causa problemas neurológicos.

2. Desarrollo psicomotor extremadamente anormal en los primeros 6 meses de vida - Criterios de apoyo para SR atípico:

1. Trastornos respiratorios cuando está despierto.

2. Bruxismo cuando está despierto.

3. Patrón de sueño alterado.

4. Tono muscular anormal.

5. Trastornos vasomotores periféricos.

6. Escoliosis/cifosis.

7. Retraso del crecimiento.

8. Manos y pies fríos.

9. Risa/gritos inapropiados.

10. Respuesta disminuida al dolor.

11. Comunicac ión visual intensa: «señalar con el ojo».

En el curso de la condición se definen cuatro estadios:

I. Detención del neurodesarrollo (6-18 meses).

II. Regresión o deterioro rápido (1-4 años).

III. Pseudoestacionario (2-10 años).

IV. Deterioro motor tardío (> 10 años).

En el estadio I se pueden observar hipotonía, retraso en el desarrollo motor, pérdida del contacto visual, retorcimiento de las manos y episodios de respiración apnéustica e hiperventilación ${ }^{6,7}$. El estadio II se presenta con un deterioro neurológico rápido; este estadio puede durar semanas o meses, y se caracteriza por cambios en el comportamiento, como pérdida de 
interacción social y de habilidades comunicativas, pérdida de lenguaje, y comienzan los movimientos estereotípicos de las manos (retorcerlas, aplaudir, lavarse o movimientos de la mano a la boca). Los pacientes también pueden tener anomalías de la respiración, crisis convulsivas y trastornos del sueño. En el estadio III puede haber una leve mejoría en el comportamiento y en las habilidades de comunicación; sin embargo, el deterioro mental y los movimientos estereotípicos de las manos continúan. Se pueden agregar rigidez, bruxismo y movimientos involuntarios de la lengua, crisis convulsivas y dificultad para la alimentación. En el estadio IV, la frecuencia de las crisis convulsivas puede disminuir, mientras que los problemas motores aumentan, incluidos la hipertonía, la distonía y la rigidez, e incluso algunos pacientes dejan de caminar ${ }^{6,7}$.

Dentro del abordaje inicial en un paciente con sospecha de SR, los diagnósticos diferenciales que se deben considerar son el síndrome de Angelman, la encefalopatía epiléptica infantil temprana y la variante congénita de $\mathrm{SR}^{8}$.

Las variantes patogénicas del gen MECP2 causan hasta el $95 \%$ de los casos de SR típico ${ }^{9}$. La confirmación diagnóstica de SR se realiza mediante la secuenciación del gen y el análisis de deleciones o duplicaciones. Sin embargo, no se han identificado mutaciones del gen MECP2 mediante secuenciación en el $3-5 \%$ de los pacientes que cumplen con los criterios de diagnóstico clínico de SR típico y en el $50-70 \%$ de los casos atípicos ${ }^{10}$.

El objetivo del presente reporte es describir dos casos de SR, uno de ellos con una variante en MECP2 no descrita previamente, y realizar una revisión de la literatura para el diagnóstico del SR.

\section{Casos clínicos}

\section{Caso 1}

Acude a la consulta un paciente de sexo femenino de 5 años, referida por neurología pediátrica, con microcefalia y regresión del desarrollo psicomotor a partir de los 3 años de edad. La paciente es producto de la segunda gesta de progenitores no consanguíneos, sin antecedentes heredofamiliares de relevancia. La madre refirió cursar un embarazo normoevolutivo. La paciente nació a las 38 semanas de gestación por vía cesárea iterativa, sin complicaciones perinatales y somatometría dentro de parámetros normales.

En la exploración física se encontraron los signos vitales dentro de los parámetros normales, con peso de
$10.8 \mathrm{~kg}$ (5.1 desviaciones estándar [DE] por debajo de la media para su sexo y edad en la población mexicana), talla de $104 \mathrm{~cm}$ (en el percentil 20) y perímetro cefálico de $47 \mathrm{~cm}$ (3.1 DE por debajo de la media). La paciente cursaba con ataxia troncal, bruxismo y movimientos estereotipados de manos y boca. Se diagnosticó clínicamente SR típico con datos correspondientes al estadio III, ya que había presentado un periodo de regresión seguido por un periodo de estabilización y cumplía todos los criterios mayores ${ }^{10}$. Se inició un abordaje diagnóstico integral. El electroencefalograma mostró un foco irritativo de puntas y ondas agudas localizado sobre la región hemisférica izquierda de predominio frontal. En la resonancia magnética cerebral se observó un incremento en la amplitud de la cisterna prepontina y prebulbar, hipoplasia del cuerpo calloso y el resto de las estructuras del encéfalo normales para la edad de la paciente.

De acuerdo con el diagnóstico clínico de SR, se solicitó la secuenciación del gen MECP2. Tras la obtención del consentimiento de los padres, se extrajo ADN de sangre periférica, se realizó la amplificación de los exones 3 y 4 de $M E C P 2$ por reacción en cadena de la polimerasa y se llevó a cabo una secuenciación automatizada directa (tipo Sanger con Big Dye Terminator Cycle sequencing kit). Se realizó la secuenciación de los exones 3 y 4 debido a que la mayoría de las variantes patogénicas se encuentran en estos exones ${ }^{11}$.

El análisis bioinformático se hizo con el programa BioEdit, lo que permitió identificar una variante en estado heterocigoto c.606delC (p.Thr203Argfs ${ }^{\star} 7$ ) (Fig. 1) que no ha sido reportada en las bases de datos de variantes del genoma humano (ClinVar, HGVD, HGMD, LOVD).

\section{Caso 2}

Acude a la consulta un paciente de sexo femenino de 17 años, referida para evaluación por síndrome dismórfico. La paciente es producto de la tercera gesta de progenitores no consanguíneos, la madre con antecedente perinatal de un aborto espontáneo del primer trimestre y un óbito de 28 semanas de gestación con malformación no especificada en la región cervicodorsal. Nació a las 38 semanas de gestación vía parto, sin complicaciones perinatales y somatometría dentro de los parámetros normales. A los 2 años de edad inició con regresión del desarrollo psicomotor, con pérdida del lenguaje y de la marcha. Previo a la valoración por genética fue diagnosticada y tratada como espectro autista. Es dependiente de silla de ruedas desde los 8 


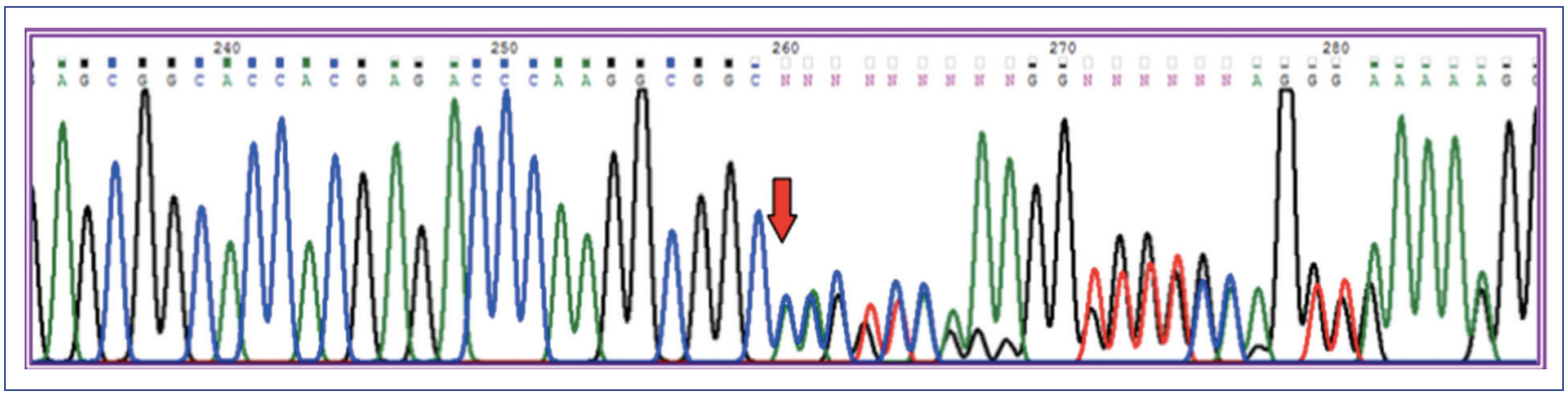

Figura 1. Electroferograma parcial correspondiente al exón 4 del gen MECP2.

años. Actualmente muestra una discapacidad intelectual grave.

En la exploración física se encontraron los signos vitales dentro de los parámetros normales. Su peso es de $25 \mathrm{~kg}$ (11 DE por debajo de la media para su sexo y edad en población mexicana), la talla de $139 \mathrm{~cm}$ (3.7 DE por debajo de la media) y el perímetro cefálico de $49 \mathrm{~cm}$ (5.6 DE por debajo de la media). Se observan escoliosis grave, ataxia y movimientos estereotipados en las manos. Se diagnosticó clínicamente SR típico, ya que había cursado con un periodo de regresión seguido de un periodo de estabilización y cumplía todos los criterios mayores que corresponden al estadio IV del SR ${ }^{10}$. En el abordaje diagnóstico, el electroencefalograma mostró un fondo lento para la edad en rango theta, así como paroxismos de ondas agudas y punta aguda en la región frontal bilateral. En la resonancia magnética cerebral solo se observó microcefalia, sin identificar otras anomalías. De acuerdo con el diagnóstico clínico de SR, se solicitó la secuenciación de nueva generación del gen MECP2. Tras la autorización de los padres y la obtención del consentimiento informado, se extrajo ADN de sangre periférica, se amplificaron los cuatro exones y regiones flanqueantes del gen MECP2, y se llevó a cabo la secuenciación de ADN y electroforesis capilar. El análisis bioinformático se realizó con el programa BioEdit por comparación con la secuencia y se identificó una variante patogénica en estado heterocigoto c.880C >T (p.Arg294*) en MECP2, que resulta en la sustitución de una arginina en la posición 880 por un codón de paro. Este cambio ya ha sido descrito previamente como una variante patogénica asociada al $\mathrm{SR}^{12}$. Asimismo, se encontró una variante de nucleótido sencillo c.1233C>T(p.Ser411Ser) rs3027928, la cual se interpreta sin relevancia clínica.

\section{Discusión}

El SR se origina principalmente por mutaciones de pérdida de función en el gen $M E C P 2$, el cual codifica para la proteína MeCP2. Esta proteína de expresión pleiotrópica se encuentra altamente expresada en diferentes áreas del cerebro ${ }^{13}$, con función de activador y represor transcripcional, así como de regulación postranscripcional de genes relacionados con la actividad sináptica ${ }^{7,14}$. Se ha postulado que MeCP2 también contribuye al destino celular en la especificación y la migración neuronales durante el desarrollo cerebral, tanto en el periodo embrionario como en el posnatal ${ }^{15}$. El mecanismo neuropatológico que lleva a la regresión del neurodesarrollo aún no se comprende del todo. Sin embargo, se postula que la regresión no resulta de la neurodegeneración, sino que surge de una función neuronal alterada ${ }^{16}$.

El cuadro clínico asociado al SR se define por los criterios consensados por Neul et $\mathrm{al}^{6}$. La confirmación diagnóstica se realiza mediante secuenciación del gen MECP2. Sin embargo, esta metodología detecta del 90-95\% de los casos de SR típico, mientras que el análisis de deleciones o duplicaciones detecta únicamente del $5-10 \%{ }^{9}$. Por otro lado, no es posible identificar mutaciones en el gen MECP2 en el 3-5\% de los pacientes que cumplen con los criterios clínicos diagnósticos de SR típico y en el $50-70 \%$ de los casos de $S R$ atípico ${ }^{10}$. Se han reportado más de 900 variantes en el gen MECP2 ${ }^{11}$, pero existen ocho hotspots (p.Arg106Trp, p.Arg133Cys, p.Thr158Met, p.Arg168*, p.Arg255, p.Arg270, p.Arg294, y p.Arg306Cys) que constituyen más del $60 \%$ de los casos documenta$\operatorname{dos}^{10,17}$. En el 5\% de los pacientes con SR no se logra identificar variantes patogénicas en MECP2, por lo que se han propuesto otros mecanismos moleculares causados por variantes patogénicas en WDR45, CTNNB1, CDKL5 y FOXG177,18. Asimismo, se han encontrado variantes en múltiples genes causantes de un síndrome similar al SR: STXBP1, TCF4, SCN2A, MEF2C y SHANK3, entre otros ${ }^{19}$.

La variante encontrada en el primer caso descrito, c.606delC (p.Thr203Argfs ${ }^{\star} 7$ ), no ha sido reportada 
previamente en bases de datos de variantes del genoma humano (ClinVar, HGVD, HGMD, LOVD). De acuerdo con los lineamientos establecidos por el American College of Medical Genetics and Genomics, se clasifica como una variante patogénica con un grado de evidencia muy sólido (PVS1), ya que se trata de una variante nula en el gen cuya pérdida de función resultante es un mecanismo conocido del $\mathrm{SR}^{20}$. El análisis predice que esta variante impide la síntesis de la proteína MeCP2 a partir de los dominios de represión transcripcional y de señal de localización nuclear. La presencia de esta variante del gen MECP2 en estado heterocigoto más el curso clínico de la paciente se consideran como evidencia suficiente para establecer el diagnóstico de SR.

La heterogeneidad clínica y genética del espectro de SR supone un reto diagnóstico. En la actualidad, con las nuevas técnicas de secuenciación y de bioinformática, más pacientes pueden contar con un diagnóstico genético certero, lo cual es primordial para un adecuado asesoramiento genético, opciones de tratamiento y perspectiva a futuro de los pacientes ${ }^{19}$.

Debido a que el $99.5 \%$ de los casos de SR son casos simplex, en los cuales existe una variante patogénica de novo en el caso índice, el riesgo de recurrencia para progenitores sanos es muy similar al de la población general, a pesar de que se han reportado muy pocos casos en la literatura de mosaicismo germinal ${ }^{21}$.

En cuanto a una relación genotipo-fenotipo, ha sido inconsistente y se postula que podría deberse al patrón de inactivación del cromosoma X. Pocas variantes patogénicas cuentan con una relación genotipo-fenotipo bien definida ${ }^{22}$, por lo que se ha propuesto que todos los pacientes con fenotipo de SR o similar a SR sean englobados en un espectro con heterogeneidad genética $^{19}$.

Resulta primordial considerar una etiología genética en los pacientes con trastornos del neurodesarrollo, e incluso con trastornos del espectro autista ${ }^{23}$. Para los profesionales de la salud que evalúan pacientes con compromiso del neurodesarrollo podría ser relativamente sencillo sospechar el diagnóstico de SR. Sin embargo, el SR atípico puede suponer un desafío. Es importante confirmar la variante patogénica para brindar un asesoramiento genético integral, que comprende exponer la evolución natural de la patología y el pronóstico, e informar a los progenitores sobre los riesgos reproductivos, para que ellos puedan tomar una decisión informada relacionada con su futuro reproductivo y el manejo integral del paciente.
Los individuos con diagnóstico de SR requieren un seguimiento interdisciplinario por subespecialidades pediátricas como neurología, gastroenterología, neumología, cardiología, medicina física y rehabilitación, paidopsiquiatría y terapia familiar psicológica con evaluaciones periódicas. El objetivo primario del manejo longitudinal es disminuir el impacto de las complicaciones y del compromiso neuromuscular secundario, siempre en busca del máximo beneficio y la mejor calidad de vida de estos pacientes y sus familias.

\section{Responsabilidades éticas}

Protección de personas y animales. Los autores declaran que para esta investigación no se han realizado experimentos en seres humanos ni en animales.

Confidencialidad de los datos. Los autores declaran que han seguido los protocolos de su centro de trabajo sobre la publicación de datos de pacientes.

Derecho a la privacidad y consentimiento informado. Los autores han obtenido el consentimiento informado de los pacientes o individuos referidos en el artículo. Este documento obra en poder del autor de correspondencia.

\section{Conflicto de intereses}

Los autores declaran no tener ningún conflicto de intereses.

\section{Financiamiento}

Ninguno.

\section{Agradecimientos}

A los pacientes y familiares.

\section{Bibliografía}

1. Rett A. [On a unusual brain atrophy syndrome in hyperammonemia in childhood]. Wien Med Wochenschr. 1966;116:723-6.

2. Amir RE, Van den Veyver IB, Wan M, Tran CQ, Francke U, Zoghbi HY. Rett syndrome is caused by mutations in X-linked MECP2, encoding methyl-CpG-binding protein 2. Nat Genet. 1999;23:185-8.

3. Tillotson R, Selfridge J, Koerner MV, Gadalla KKE, Guy J, De Sousa D, et al. Radically truncated MeCP2 rescues Rett syndrome-like neurological defects. Nature. 2017;550:398-401.

4. Ip JPK, Mellios N, Sur M. Rett syndrome: insights into genetic, molecular and circuit mechanisms. Nat Rev Neurosci. 2018;19:368-82.

5. Neul JL. The relationship of Rett syndrome and MECP2 disorders to autism. Dialogues Clin Neurosci. 2012;14:253-62.

6. Neul JL, Kaufmann WE, Glaze DG, Christodoulou J, Clarke AJ, Bahi-Buisson N, et al. Rett syndrome: revised diagnostic criteria and nomenclature. Ann Neurol. 2010;68:944-50.

7. Einspieler C, Marschik PB. Regression in Rett syndrome: developmental pathways to its onset. Neurosci Biobehav Rev. 2019;98:320-32. 
8. Kaur S, Christodoulou J. MECP2 disorders. En: Adam MP, Ardinger HH, Pagon RA, et al., editores. GeneReviews $®$. Seattle (WA): University of Washington, Seattle; 2001. p. 1993-2020.

9. Philippe C, Villard L, De Roux N, Raynaud M, Bonnefond JP, Pasquier L, et al. Spectrum and distribution of MECP2 mutations in 424 Rett syndrome patients: a molecular update. Eur J Med Genet. 2006;49:9 18.

10. Neul JL, Fang P, Barrish J, Lane J, Caeg EB, Smith EO, et al. Specific mutations in methyl-CpG-binding protein 2 confer different severity in Rett syndrome. Neurology. 2008;70:1313-21.

11. Summary of MECP2 mutations. Summary by frequency. Rettsyndrome. org. (Consultado el 22 de julio de 2020.) Disponible en: http://mecp2.chw. edu.au/mecp2_summary_mutations.php?sel=freq

12. Cheadle JP, Gill H, Fleming N, Maynard J, Kerr A, Leonard H, et al. Long-read sequence analysis of the MECP2 gene in Rett syndrome patients: correlation of disease severity with mutation type and location. Hum Mol Genet. 2000;9:1119-29.

13. Horvath PM, Monteggia LM. MeCP2 as an activator of gene expression. Trends Neurosci. 2018;41:72-4.

14. Chahrour M, Jung SY, Shaw C, Zhou X, Wong ST, Qin J, et al. MeCP2, a key contributor to neurological disease, activates and represses transcription. Science. 2008;320:1224-9.

15. Feldman D, Banerjee A, Sur M. Developmental dynamics of Rett syndrome. Neural Plasticity. 2016;2016:6154080.
16. Zoghbi HY. Rett syndrome and the ongoing legacy of close clinical observation. Cell. 2016:167;293-7.

17. Percy AK, Lane J, Annese F, Warren H, Skinner SA, Neul JL. When Rett syndrome is due to genes other than MECP2. Transl Sci Rare Dis. 2018;3:49-53.

18. Naidu S, Johnston MV. Neurodevelopmental disorders: clinical criteria for Rett syndrome. Nat Rev Neurol. 2011;7:312-4.

19. Vidal S, Xiol C, Pascual-Alonso A, O'Callaghan M, Pineda M, Armstrong J. Genetic landscape of Rett syndrome spectrum: improvements and challenges. Int J Mol Sci. 2019;20:3925.

20. Richards S, Aziz N, Bale S, Bick D, Das S, Gastier-Foster J, et al. Standards and guidelines for the interpretation of sequence variants: a joint consensus recommendation of the American College of Medical Genetics and Genomics and the Association for Molecular Pathology. Genet Med. 2015;17:405-24.

21. Zhang Q, Yang X, Wang J, Li J, Wu Q, Wen Y, et al. Genomic mosaicism in the pathogenesis and inheritance of a Rett syndrome cohort. Genet Med. 2019;21:1330-8.

22. Charman T, Neilson TC, Mash V, Archer H, Gardiner MT, Knudsen GP et al. Dimensional phenotypic analysis and functional categorisation of mutations reveal novel genotype-phenotype associations in Rett syndrome. Eur J Hum Genet. 2005;13:1121-30.

23. Yoo H. Genetics of autism spectrum disorder: current status and possible clinical applications. Exp Neurobiol. 2015;24:257-72. 